\title{
Clinical Profile and Treatment Management of Heart Failure with Preserved Systolic Function in Rural Setting of India
}

\author{
Tom Devasia1, Sunil Datta Nandibandi', Rama Bhat ${ }^{2}$, Hashir Kareem¹, Ashok Thakkar3 \\ ${ }^{1}$ Department of Cardiology, Kasturba Medical College \& Hospital, Manipal, India \\ ${ }^{2}$ Department of Medicine, Kasturba Medical College \& Hospital, Manipal, India \\ ${ }^{3}$ Department of Clinical Trials, Sahjanand Medical Technologies Pvt. Ltd., Surat, India \\ Email: tomdevasia@hotmail.com
}

Received 31 December 2013; revised 28 January 2014; accepted 27 February 2014

Copyright @ 2014 by authors and Scientific Research Publishing Inc.

This work is licensed under the Creative Commons Attribution International License (CC BY).

http://creativecommons.org/licenses/by/4.0/

(c) (i) Open Access

\section{Abstract}

Background: In recent decades, the hospital admission due to heart failure with normal ejection fraction (HFnEF) or diastolic heart failure has increased particularly in elderly patients. However, sufficient data regarding prevalence, etiologies and treatment of diastolic heart failure are not available for Indian population. So, we carried out an observational study to determine clinical profile and medical therapy for patients experiencing diastolic heart failure. Methods: This was prospective observational study carried out in rural area of India for the period of 12 months. All the patients diagnosed with heart failure with normal ejection fraction were included in the study. If the patient was having severe anemia (hemoglobin $<8.00 \mathrm{~g} / \mathrm{dl}$ ), hemodynamically significant valvular disease, prosthetic valve replacement, and ventricular pacemaker, they were excluded. Results: A total of 53 patients diagnosed with HFnEF were included in the study. There were 24 male patients. Hypertension, CAD and diabetes mellitus were present in 33, 24 and 16 patients respectively. 18 patients developed severe diastolic dysfunction and more common in female as compared to male $(37.9 \%$ vs. $33.3 \%)$. Most frequently observed clinical feature was tachycardia ( $96 \%$ cases) followed by pedal edema (86\%). The patients were treated according to underlying cause. Conclusions: Diastolic heart failure is more common in elderly patients. In Indian population, diastolic heart failure has been associated with hypertension, diabetes mellitus and coronary artery diseases in most of the cases.

\section{Keywords}

Diastolic Heart Failure; Epidemiology; Heart Failure; Normal Ejection Fraction

How to cite this paper: Devasia, T., Nandibandi, S.D., Bhat, R., Kareem, H. and Thakkar, A. (2014) Clinical Profile and Treatment Management of Heart Failure with Preserved Systolic Function in Rural Setting of India. International Journal of Clinical Medicine, 5, 171-176. http://dx.doi.org/10.4236/ijcm.2014.55030 


\section{Introduction}

Epidemiological studies have revealed that $1.5 \%$ to $2 \%$ population experience heart failure (HF) and it is the main reason for hospital admission of elderly patients [1]. It has been estimated that the prevalence increases to $6 \%-10 \%$ in patients over 65 years of age [2] [3]. The survival after HF has improved due to advancement in treatment. However, HF is one of the leading causes of death, approximately 30,000 deaths per year [4].

Recent studies have indicated that more than half patients diagnosed with HF even though ejection fraction (EF) is normal or near normal [5] [6]. This clinical condition is termed as "heart failure with normal ejection fraction" (HFnEF) or "diastolic heart failure" (DHF). It is characterized by the evidence of diastolic dysfunction (e.g., impaired LV relaxation, abnormal left ventricular filling and elevated filling pressure). There has been substantial progress in the treatment of systolic heart failure (SHF). However, very little progress has been made in the management of DHF. The mortality due to DHF is $8 \%$ to $9 \%$ per year which is about the half of the SHF but the morbidity, hospitalisation rates and healthcare costs per patient are almost similar to those of SHF [7].

The common risk factors associated with DHF i.e. hypertension, diabetes and coronary artery diseases are more prevalent in India [6] [8]. Due to higher propensity for these risk factors, there is a possibility that the burden of DHF is likely to be higher in India. As the exact prevalence and incidence of DHF are not known, it indicates a need to carry out the study which gives us an idea regarding the prevalence, aetiology, morbidity and mortality pattern of this study. So, we have carried out the study to obtain the clinical profile of the patients experiencing DHF along with aetiology, pharmacological treatments and short-term outcomes.

\section{Methods}

\subsection{Study Design and Patient Population}

This prospective observational study was carried out at Kasturba Hospital, Manipal between January 2010 and February 2012. The protocol of the study was approved by institutional ethics committee of the hospital before the initiation of the study. The informed consents were obtained from all the patients enrolled in the study.

All the patients, newly diagnosed with HF and having normal EF, were enrolled in the study according to the inclusion and exclusion criteria. Inclusion criteria: If the patients admitted to medicine or cardiology departments of the hospital with clinical evidence of HF according to Framingham's criteria [9] along with normal EF (EF $>50 \%$ ) were included in the study. Exclusion criteria: the patients who had 1) severe anemia (hemoglobin < $8.00 \mathrm{~g} / \mathrm{dl}$ ), 2) hemodynamically significant valvular disease, 3) prosthetic valve replacement, 4) ventricular pacemaker.

The demographic detail of the patients was collected from the medical records of the patient using pre-defined case-record form. Each patient underwent thorough physical examinations including electrocardiogram, echocardiography, chest radiology and specific laboratory tests (complete blood count, renal function test). Treatment was started according to the underlying condition. Patients were followed till discharge from the hospital.

The patients were diagnosed with renal dysfunction if creatinine level was $>1.4 \mathrm{mg} / \mathrm{dl}$. The patients were classified into two groups (mild-moderate diastolic dysfunction and severe diastolic dysfunction) based on the mitral inflow early-diastole (E) and atrial contraction (A) velocities and deceleration time as follow:

- If $\mathrm{E} / \mathrm{A}<1.5$ and Deceleration time $>140 \mathrm{~ms}$, the patients were diagnosed with mild-moderate diastolic dysfunction;

- If E/A > 1.5 and Deceleration time $<140 \mathrm{~ms}$, the patients were diagnosed with severe diastolic dysfunction.

\subsection{Statistical Analysis}

Statistical analysis was performed using Statistical Package for Social Sciences (SPSS; Chicago, IL, USA) program, version 15 . Continuous data are expressed as mean \pm standard deviation.

\section{Results}

\subsection{Baseline Demographics and Patient Characteristics}

During the study period a total 53 patients diagnosed with HFnEF were evaluated. The demographic detail of the patients enrolled in the study is shown in Table 1. There were 29 female patients (mean age 64.5) as com- 
Table 1. Baseline clinical and demographic characteristics.

\begin{tabular}{cc}
\hline $\begin{array}{c}\text { Total patients } \\
\text { Mean age }\end{array}$ & N (\%) \\
Male & 53 \\
Female & $63.5 \pm 14.7$ \\
& \\
Gypertension & $24(45 \%)$ \\
Risk factors & $29(55 \%)$ \\
Cardiovascular diseases & \\
Diabetes mellitus & $33(62.3 \%)$ \\
Valvular heart disease & $24(45.3 \%)$ \\
Atrial fibrillation & $16(30.2 \%)$ \\
Sepsis & $11(20.8 \%)$ \\
Hypertrophic cardiomyopathy & $5(9.4 \%)$ \\
\end{tabular}

pared to 24 male patients (mean age 64.3).

Figure 1 shows age wise distribution of patients experienced HFnEF. Hypertension, coronary artery disease (CAD) and diabetes mellitus, the risk factors for HFnEF, was present in 33, 24 and 16 patients respectively. Renal dysfunction (creatinine $>1.4 \mathrm{mg} / \mathrm{dl}$ ) was observed in 10 patients. Severe diastolic dysfunction was more common in females $(\mathrm{n}=11,37.9 \%)$ than males $(\mathrm{n}=8,33.3 \%)$.

\subsection{Clinical Features of Patients}

Tachycardia was the commonest clinical sign (96\%) among the patients with diastolic dysfunction. Others were pedal edema (86\%), raised JVP (63\%) and pulmonary edema (46\%). It has been observed that patients with severe diastolic dysfunction were more likely to experience pulmonary edema as compared to those with mild to moderate diastolic dysfunction (63.2\% vs. $41.2 \%)$.

\subsection{ECG Pattern of Patients}

Tachycardia-abnormal rhythm indicating heart failure was frequently observed (96\%) followed by T-wave inversion (58\%) and ST-segment depression (41\%). Atrial fibrillation and P-pulmonale were observed in 38\% and $32 \%$ cases.

\subsection{Pharmacological Treatment}

The medical therapy initiated to the patients diagnosed with HFnEF has been shown in Table 2.

\subsection{Clinical Outcomes}

During hospitalization, out of 53 patients enrolled in the study, total 3 patients died. Two patients died as a result of sudden cardiac arrest whereas complicated pneumonia along with sepsis and multi-organ dysfunction was the reason of one death.

\section{Discussions}

Heart failure with normal ejection fraction is a challenging problem for clinicians and patients. Treatment of HFnEF has been exclusively to treat the underlying cause. i.e. $\beta$-blockers can improve ventricular function in patients with hypertrophic cardiomyopathy [10]. But as we unfold the associations and pathogenesis, new modalities of therapy which address the diastolic dysfunction may come in future. Hence there is a need for studies that establish the clinical profile and outcome in patients with heart failure with normal ejection fraction.

A total of 53 patients experienced HFnEF during the study period were enrolled. The results of the study showed that females (55\%) were more prone to develop HFnEF as compared to male patients. The result of our study is similar to that of the study conducted by Maestre et al. [5] in which 64.4\% patients, who experienced 


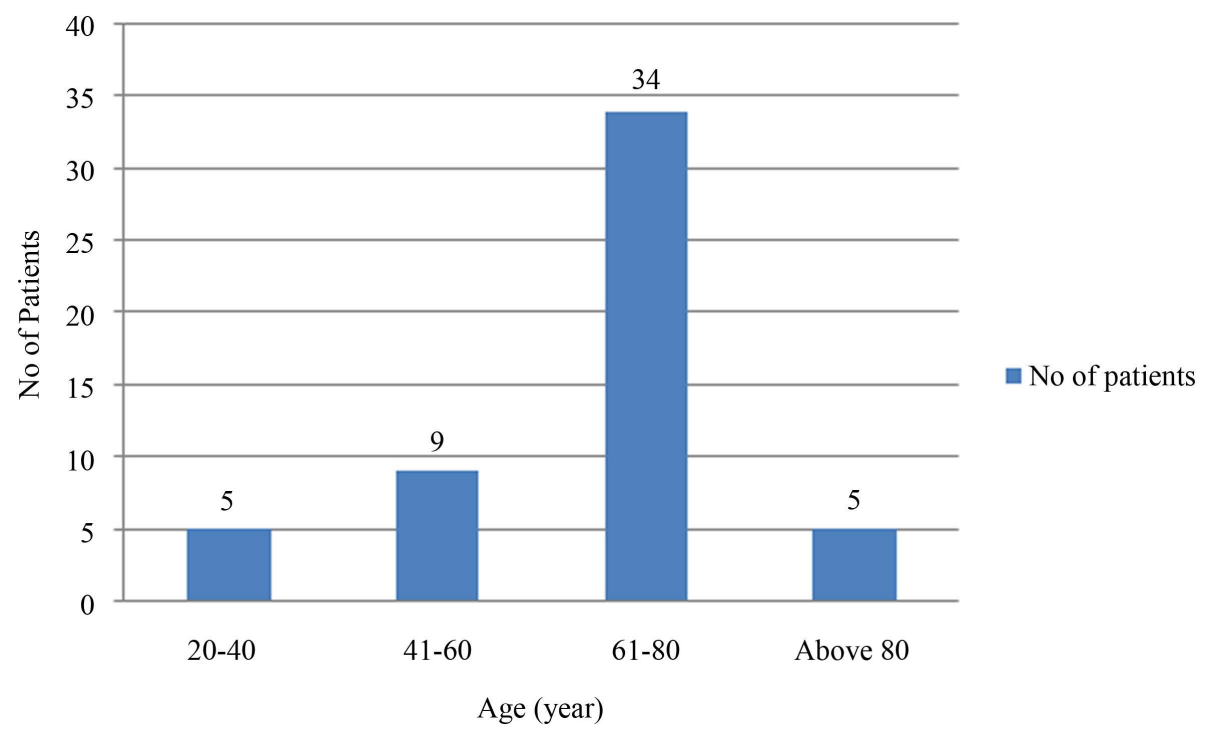

Figure1. Age wise distribution of patients experienced HFnEF.

Table 2. Treatment of patient experienced HFnEF.

\begin{tabular}{cc}
\hline Medical therapy prescribed to the patients & N (\%) \\
Diuretics & $49(92 \%)$ \\
Anti-platelets & $36(68 \%)$ \\
Statins & $33(62 \%)$ \\
ACE-inhibitors & $31(58 \%)$ \\
Amlodipine & $18(34 \%)$ \\
Nitrates & $6(12 \%)$ \\
p-blockers & $4(8 \%)$ \\
Digoxin & $3(6 \%)$ \\
\hline
\end{tabular}

HFnEF, were female. Similar result was obtained in the studies carried out by Masoudi et al. [11] and Yancy et al. [12]. Other cross-sectional studies also showed similar results [13] [14]. The echocardiographic evaluation of patients in our study also indicated that severe diastolic dysfunction is more common in female $(37.9 \%$ vs. 33.3\%).

The epidemiological studies have shown that HFnEF is the disease of elder population. In our study also out of 53 patients, 39 (73.6\%) patients were elder. Several community based studies demonstrated similar finding [15] [16].

The spectrum of aetiologies found in our observational studies was hypertension (62.3\%), cardiovascular disease $(45.3 \%)$ followed by diabetes mellitus (30.2\%). Other aetiologies were valvular heart disease, abnormal heart rhythm (atrial fibrillation), sepsis and hypertrophic cardiomyopathy. Klapholz et al. in their multicenter registry demonstrated similar results i.e. hypertension in 78\%, CAD in 43\% and diabetes in $46 \%$ of cases [17]. Dubourg et al. had also reported similar results with hypertension (61.2\%) being most commonly associated risk factor followed by CAD (47.1\%) and diabetes (24.6\%) [6].

The treatment of HFnEF remains empiric since limited trial data are available [18]. The primary goals of the treatment are to improve symptoms, reverse disease progression, reduce hospitalisation and enhance life expectancy. Majority of the patients, in our study, came with history of exertional dyspnea and pedal edema. All these patients were treated according to the underlying cause those were with antihypertensives (ACE inhibitors, $\beta$-blockers), diuretics to decrease the volume overload, ionotropic agents (digoxin), nitrates, calcium channel blockers, statin, and antiplatelet agents.

\section{Conclusion}

This study provides comprehensive clinical profile, current management, and prognosis of patients with new onset 
of DHF. We can conclude that DHF was more prevalent in female and in elderly patients particularly after 60 years. The most common etiologies for DHF were hypertension, coronary artery disease and diabetes mellitus.

\section{References}

[1] Cowie, M., Mosterd, A., Wood, D., Deckers, J., Poole-Wilson, P., Sutton, G. and Grobbee, D. (1997) The Epidemiology of Heart Failure. European Heart Journal, 18, 208-225. http://dx.doi.org/10.1093/oxfordjournals.eurheartj.a015223

[2] Mosterd, A.H., De Bruyne, M., Deckers, J., Linker, D., Hofman, A. and Grobbee, D. (1999) Prevalence of Heart Failure and Left Ventricular Dysfunction in the General Population; The Rotterdam Study. European Heart Journal, 20, 447-455. http://dx.doi.org/10.1053/euhj.1998.1239

[3] Ho, K.K., Pinsky, J.L., Kannel, W.B. and Levy, D. (1993) The Epidemiology of Heart Failure: The Framingham Study. Journal of the American College of Cardiology, 22, 6A-13A. http://dx.doi.org/10.1016/0735-1097(93)90455-A

[4] Levy, E. (2000) Le coût Économique de l'Insuffisance Cardiaque. Archives des Maladies du Coeur et des VaisseauxPratique, 86, 9.

[5] Maestre, V.G., Gallego, J., García, M., García de Burgos, F. and Martín-Hidalgo, A. (2009) Prediction Clinical Profile to Distinguish between Systolic and Diastolic Heart Failure in Hospitalized Patients. European Journal of Internal Medicine, 20, 313-318. http://dx.doi.org/10.1016/j.ejim.2008.09.001

[6] Dubourg, O., Gueret, P., Beauchet, A., Nisse-Durgeat, S. and Ducardonnet, A. (2008) Focale: Study of Systolic and Diastolic Heart Failure in a French Elderly Population. International Journal of Cardiology, 124, 188-192. http://dx.doi.org/10.1016/j.ijcard.2007.01.050

[7] Aurigemma, G.P. and Gaasch, W.H. (2004) Diastolic Heart Failure. New England Journal of Medicine, 351, 10971105. http://dx.doi.org/10.1056/NEJMcp022709

[8] Reddy, S., Bahl, A. and Talwar, K. (2010) Congestive Heart Failure in Indians: How Do We Improve Diagnosis \& Management? The Indian Journal of Medical Research, 132, 549.

[9] Yturralde, R.F. and Gaasch, W.H. (2005) Diagnostic Criteria for Diastolic Heart Failure. Progress in Cardiovascular Diseases, 47, 314-319. http://dx.doi.org/10.1016/j.pcad.2005.02.007

[10] Hess, O.M., Murakami, T. and Krayenbuhl, H.P. (1987) Influence of Verapamil on Diastolic Left Ventricular Function in Myocardial Hypertrophy of Different Origin. Zeitschrift für Kardiologie, 76, 82-86.

[11] Masoudi, F.A., Havranek, E.P., Smith, G., Fish, R.H., Steiner, J.F., Ordin, D.L. and Krumholz, H.M. (2003) Gender, Age, and Heart Failure with Preserved Left Ventricular Systolic Function. Journal of the American College of Cardiology, 41, 217-223. http://dx.doi.org/10.1016/S0735-1097(02)02696-7

[12] Yancy, C.W., Lopatin, M., Stevenson, L.W., De Marco, T. and Fonarow, G.C. (2006) Clinical Presentation, Management, and In-Hospital Outcomes of Patients Admitted with Acute Decompensated Heart Failure with Preserved Systolic Function: A Report from the Acute Decompensated Heart Failure National Registry (ADHERE) Database. Journal of the American College of Cardiology, 47, 76-84. http://dx.doi.org/10.1016/j.jacc.2005.09.022

[13] Chen, H.H., Lainchbury, J.G., Senni, M., Bailey, K.R. and Redfield, M.M. (2002) Diastolic Heart Failure in the Community: Clinical Profile, Natural History, Therapy, and Impact of Proposed Diagnostic Criteria. Journal of Cardiac Failure, 8, 279-287. http://dx.doi.org/10.1054/jcaf.2002.128871

[14] Acikel, S., Akdemir, R., Kilic, H., Yesilay, A.B., Dogan, M. and Cagirci, G. (2010) Diastolic Heart Failure in Elderly: The Prognostic Factors and Interventions Regarding Heart Failure with Preserved Ejection Fraction. International Journal of Cardiology, 138, 311-313. http://dx.doi.org/10.1016/j.ijcard.2008.06.068

[15] Pernenkil, M., Vinson, M., Janice, M., Shah, M., Atul, S., Beckham, R., Wittenberg, R., Rich, M. and Michael, W. (1997) Course and Prognosis in Patients $\geq 70$ Years of Age with Congestive Heart Failure and Normal versus Abnormal Left Ventricular Ejection Fraction. The American Journal of Cardiology, 79, 216-219. http://dx.doi.org/10.1016/S0002-9149(96)00719-9

[16] McAlister, F.A., Teo, K.K., Taher, M., Montague, T.J., Humen, D., Cheung, L., Kiaii, M., Yim, R. and Armstrong, P.W. (1999) Insights into the Contemporary Epidemiology and Outpatient Management of Congestive Heart Failure. American Heart Journal, 138, 87-94. http://dx.doi.org/10.1016/S0002-8703(99)70251-6

[17] Klapholz, M., Maurer, M., Lowe, A.M., Messineo, F., Meisner, J.S., Mitchell, J., Kalman, J., Phillips, R.A., Steingart, R. and Brown, E.J. (2004) Hospitalization for Heart Failure in the Presence of a Normal Left Ventricular Ejection Fraction Results of the New York Heart Failure Registry. Journal of the American College of Cardiology, 43, 14321438. http://dx.doi.org/10.1016/j.jacc.2003.11.040

[18] Cohen-Solal, A. (2002) Diastolic Heart Failure: Myth or Reality? European Journal of Heart Failure, 4, 395-400. http://dx.doi.org/10.1016/S1388-9842(02)00033-8 


\section{List of Abbreviations}

\begin{tabular}{cl}
\hline ACE & Angiotensin Converting Enzyme \\
DHF & Diastolic Heart Failure \\
EF & Ejection Fraction \\
HF & Heart Failure \\
HFnEF & Heart Failure with normal Ejection Fraction \\
JVP & Jugular Venous Pressure \\
SHF & Systolic Heart Failure \\
\hline
\end{tabular}

